\title{
A NOTE ON THE INJECTIVITY OF ACTIONS OF COMPACT QUANTUM GROUPS ON A CLASS OF $C^{*}$-ALGEBRAS
}

\author{
DEBASHISH GOSWAMI and SOUMALYA JOARDAR \\ Indian Statistical Institute \\ 203, B. T. Road, Kolkata 700108, India \\ ORCID: 0000-0002-8405-4967 (S.J.) \\ E-mail: goswamid@isical.ac.in, soumalya.j@gmail.com
}

Dedicated to Paul F. Baum on the occasion of his eightieth birthday

\begin{abstract}
We give some sufficient conditions for the injectivity of actions of compact quantum groups on $C^{*}$-algebras. As an application, we prove that any faithful smooth action by a compact quantum group on a compact smooth (not necessarily connected) manifold is injective. A similar result is proved for actions on $C^{*}$-algebras obtained by Rieffel deformations of compact, smooth manifolds.
\end{abstract}

1. Introduction. Quantum groups are natural generalization of groups and they are used as 'generalized symmetry objects' in mathematics and physics. The pioneering work by Drinfeld and Jimbo ([6], [5], [13, [12]) and others gave the formulation of quantum groups in the algebraic setting as Hopf algebras typically obtained by deforming the universal enveloping algebras of semisimple Lie algebras. This led to a deep and successful theory having connections with physics, knot theory, number theory, representation theory etc. On the other hand, S. L. Woronowicz (see, e.g. [22]) approached it from a point of view of harmonic analysis on locally compact groups and came up with a set

2010 Mathematics Subject Classification: 81R50, 81R60, 20G42, 58B34.

Key words and phrases: Compact quantum group, Riemannian manifold, smooth action.

D.G. partially supported by J C Bose Fellowship from D.S.T. (Govt. of India) and also acknowledges the Fields Institute, Toronto for providing hospitality for a brief stay when a small part of this work was done.

S.J. acknowledges support from CSIR.

The paper is in final form and no version of it will be published elsewhere. 
of axioms for defining compact quantum groups (CQG for short) as a generalization of compact topological groups. In this note, we will restrict ourselves to the framework of compact quantum groups only. It is natural to define quantum analogue of group action on spaces. This can be done in different ways: for the purely algebraic approach, this is defined as a co-action of Hopf algebra. In the analytic theory, there are $C^{*}$ and von Neumann algebraic notions of action. We shall consider here actions of compact quantum groups on $C^{*}$-algebras in the sense of Podleś ([17]). A subtle point about this definition is that it does not assume the injectivity of the action. We mention here that some authors (e.g. [1]) indeed prefer to include injectivity in the definition of CQG actions on $C^{*}$-algebras but this is not a universal practice. In fact, Soltan discussed in [19] several examples of non-injective actions in the sense of Podleś. On the other hand, group actions on spaces are always injective. Injectivity also follows in the algebraic setting for Hopf algebra co-actions as well as for von Neumann algebraic notion of actions of (von Neumann algebraic) quantum groups. Thus, it is an interesting and important problem to give sufficient conditions for injectivity of action of compact quantum groups in the sense of Podleś. This is the aim of this short note. We shall consider CQG actions on $C(M)$ and their Rieffel deformations where $M$ is a compact smooth manifold. Under a smoothness condition on the action on $C(M)$ (in the sense of [10]) we can prove injectivity. For the Rieffel deformation of classical manifolds, we prove injectivity under a natural analogue of smoothness and compatibility of the action with the canonical toral action.

2. Preliminaries. In this paper all Hilbert spaces are over $\mathbb{C}$ unless mentioned otherwise. For a vector space $V, V^{\prime}$ denotes its algebraic dual. $\oplus$ and $\otimes_{\text {alg }}$ will denote the algebraic direct sum and algebraic tensor product respectively. On the other hand, the minimal $C^{*}$-algebra tensor product and tensor product of Hilbert spaces as well as Hilbert modules will be denoted by $\otimes$. In particular, we consider Hilbert modules of the form $\mathcal{H} \otimes \mathcal{C}$ where $\mathcal{C}$ is a $C^{*}$-algebra. For a Hilbert $\mathcal{A}$-module $E$ we denote by $\mathcal{L}(E)$ the $C^{*}$-algebra of adjointable right $\mathcal{A}$-linear maps on $E$. We denote the $C^{*}$-algebra of bounded operators on a Hilbert space $\mathcal{H}$ by $\mathcal{B}(\mathcal{H})$ and the $C^{*}$-algebra of compact operators on $\mathcal{H}$ by $\mathcal{B}_{0}(\mathcal{H})$. $\mathrm{Sp}, \overline{\mathrm{Sp}}$ stand for the linear span and the closed linear span of elements of a vector space respectively, whereas $\operatorname{Im}(A)$ denotes the image of a linear map $A$. Given a group action $\gamma$ on a locally convex space $Z$ we denote the fixed point subspace by $Z^{\gamma}$.

We call a locally convex space Fréchet if the family of seminorms is countable (hence the space is metrizable) and the space is complete with respect to the metric given by the family of seminorms. There are many ways to equip the algebraic tensor product of two locally convex spaces with a locally convex topology. Let $E_{1}, E_{2}$ be two locally convex spaces with the corresponding families of seminorms $\left\{\|\cdot\|_{1, i}\right\}$ and $\left\{\|\cdot\|_{2, j}\right\}$ respectively. Then one wants a family $\left\{\|\cdot\|_{i, j}\right\}$ of seminorms for $E_{1} \otimes_{\text {alg }} E_{2}$ such that $\left\|e_{1} \otimes e_{2}\right\|_{i, j}=$ $\left\|e_{1}\right\|_{1, i}\left\|e_{2}\right\|_{2, j}$. The problem is that such a choice is far from unique and there is a maximal and a minimal choice giving the projective and injective tensor product respectively. Let us denote the projective tensor product by $E_{1} \hat{\otimes} E_{2}$. A Fréchet locally convex space is called nuclear if its projective and injective tensor products with any other Fréchet space coincide as a locally convex space. It is known that closed subspaces and quotients by 
closed subspaces of a nuclear Fréchet space are again nuclear. We do not go into further details of this topic here but refer the reader to [20] for a comprehensive discussion. Furthermore, if the space is a $*$-algebra then we demand that its $*$-algebraic structure is compatible with its locally convex topology, i.e. the involution $*$ is continuous and the multiplication is jointly continuous with respect to the topology. Projective and injective tensor products of two such topological $*$-algebras are again topological $*$-algebras. We shall mostly use unital $*$-algebras. Henceforth all the topological $*$-algebras will be unital unless otherwise mentioned. Consider a locally convex algebra $\mathcal{A}$ for which each of the defining seminorms, say $\|\cdot\|_{i}$, satisfies

$$
\|x y\|_{i} \leq C_{i}\|x\|_{i}\|y\|_{i}
$$

for some constant $C_{i}$ and all $x, y \in \mathcal{A}$. Then it is easy to see from the definition of the projective tensor product that the algebra multiplication map (say $m$ ) lifts to a continuous map from $\mathcal{A} \hat{\otimes} \mathcal{A}$ to $\mathcal{A}$, to be denoted by $m$ again.

We mainly need a particular class of nuclear locally convex *-algebra, which is $C^{\infty}(M)$, where $M$ is any compact smooth manifold. The natural Fréchet topology on $C^{\infty}(M)$ is given by the seminorms of the form $p^{U, K, \alpha}$,

$$
p^{U, K, \alpha}(f)=\sup _{x \in K}\left|\partial^{\alpha}(f)(x)\right|,
$$

where $K$ is a compact subset contained in the domain of some coordinate chart $\left(U,\left(x_{1}, \ldots, x_{n}\right)\right), \alpha=\left(i_{1}, \ldots, i_{k}\right)$ a multi-index and $\partial^{\alpha}=\frac{\partial}{\partial x_{i_{1}}} \ldots \frac{\partial}{\partial x_{i_{k}}}, i_{j} \in\{1, \ldots, n\}$. We can similarly define a Fréchet topology on $C^{\infty}(M, E)$, the space of smooth $E$-valued functions on $M$ for any Fréchet space $E$. We refer the reader to [10] for more details. One can verify condition (1) for the family of seminorms defining the Fréchet algebra $C^{\infty}(M, \mathcal{A})$ where $\mathcal{A}$ is a Banach algebra, by Leibniz rule.

\subsection{Compact quantum groups and their actions on $C^{*}$-algebras}

Definition 2.1. A compact quantum group (CQG for short) is a unital $C^{*}$-algebra $\mathcal{Q}$ with a coassociative coproduct $\Delta$ (see [16], [22]) from $\mathcal{Q}$ to $\mathcal{Q} \otimes \mathcal{Q}$ such that each of the linear spans of $\Delta(\mathcal{Q})(\mathcal{Q} \otimes 1)$ and that of $\Delta(\mathcal{Q})(1 \otimes \mathcal{Q})$ is norm-dense in $\mathcal{Q} \otimes \mathcal{Q}$.

A unitary representation of a CQG $\mathcal{Q}$ on a Hilbert space $\mathcal{H}$ is a unitary $U \in \mathcal{L}(\mathcal{H} \otimes \mathcal{Q})$ such that the $\mathbb{C}$-linear map $V$ from $\mathcal{H}$ to the Hilbert module $\mathcal{H} \otimes \mathcal{Q}$ given by $V(\xi)=$ $U(\xi \otimes 1)$ satisfies $(V \otimes \mathrm{id}) \circ V=(\mathrm{id} \otimes \Delta) \circ V$. Here, the map $(V \otimes \mathrm{id})$ denotes the extension of $V \otimes$ id to the completed tensor product $\mathcal{H} \otimes \mathcal{Q}$ which exists as $V$ is an isometry.

It is known that every unitary irreducible representation of a CQG is finite dimensional. Let $\Pi$ denote the set of inequivalent irreducible representations of the CQG $\mathcal{Q}$. For $\pi \in \Pi$, let $U_{\pi}=\left(\left(t_{i j}^{\pi}\right)\right)$ be the corresponding unitary element of $M_{d_{\pi}}(\mathcal{Q})$ where $d_{\pi}$ is the dimension of $\pi$. We call $t_{i j}^{\pi}$ 's the matrix coefficients of $\pi$.

Every CQG $\mathcal{Q}$ contains a canonical dense unital $*$-subalgebra $\mathcal{Q}_{0}$ of $\mathcal{Q}$ on which linear maps $\kappa$ and $\epsilon$ (called the antipode and the counit respectively) are defined making the above subalgebra a Hopf *-algebra. In fact, this is the algebra generated by the "matrix coefficients' of the (finite dimensional) irreducible unitary representations (see [16]) of the CQG. The antipode is an anti-homomorphism and also satisfies $\kappa\left(a^{*}\right)=\left(\kappa^{-1}(a)\right)^{*}$ for $a \in \mathcal{Q}_{0}$. 
It is known that there is a unique state $h$ on a CQG $\mathcal{Q}$ (called the Haar state) which is bi-invariant in the sense that $(\mathrm{id} \otimes h) \circ \Delta(a)=(h \otimes \mathrm{id}) \circ \Delta(a)=h(a) 1$ for all $a$. The Haar state need not be faithful in general, though it is always faithful on $\mathcal{Q}_{0}$ at least. The reduced CQG $\mathcal{Q}_{r}$ corresponding to $\mathcal{Q}$ is the image of $\mathcal{Q}$ in the GNS representation of $h$, i.e. $\mathcal{Q}_{r}=\pi_{r}(\mathcal{Q})$, where $\pi_{r}: \mathcal{Q} \rightarrow \mathcal{B}\left(L^{2}(h)\right)$ is the GNS representation.

Given two CQG's $\left(\mathcal{Q}_{1}, \Delta_{1}\right)$ and $\left(\mathcal{Q}_{2}, \Delta_{2}\right)$, a $*$-homomorphism $\pi: \mathcal{Q}_{1} \rightarrow \mathcal{Q}_{2}$ is said to be a CQG morphism if $(\pi \otimes \pi) \circ \Delta_{1}=\Delta_{2} \circ \pi$ on $\mathcal{Q}_{1}$. In case $\pi$ is surjective, $\mathcal{Q}_{2}$ is said to be a quantum subgroup of $\mathcal{Q}_{1}$ and we write $\mathcal{Q}_{2} \leq \mathcal{Q}_{1}$.

Definition 2.2. We say that a CQG $\mathcal{Q}$ with the coproduct $\Delta$ (co)-acts on a (unital) $C^{*}$-algebra $\mathcal{C}$ if there is a unital $*$-homomorphism $\alpha: \mathcal{C} \rightarrow \mathcal{C} \otimes \mathcal{Q}$ such that $(\alpha \otimes$ id $) \circ \alpha=$ $($ id $\otimes \Delta) \circ \alpha$, and the linear span of $\alpha(\mathcal{C})(1 \otimes \mathcal{Q})$ is norm-dense in $\mathcal{C} \otimes \mathcal{Q}$.

In Woronowicz theory, it is customary to drop 'co', and call the above co-action simply 'action' of the CQG on the $C^{*}$-algebra. Let us adopt this convention for the rest of the note.

Given an action $\alpha$ of a CQG $\mathcal{Q}$ on a unital $C^{*}$-algebra $\mathcal{C}$ and an irreducible representation $\pi \in \Pi$, we recall the idempotent operators $E_{\pi}: \mathcal{C} \rightarrow \mathcal{C}$ constructed in Theorem 1.5 of [17. It is shown in [17] that $\alpha$ leaves the image $W_{\pi}$ (say) of $E_{\pi}$ invariant and if $W_{\pi} \neq(0)$, the restriction of $\alpha$ to $W_{\pi}$ decomposes (as a vector space) into copies of the irreducible representation equivalent to $\pi$. We call $W_{\pi}$ the spectral subspace for the action $\alpha$ corresponding to the irreducible type $\pi$. Moreover, the (algebraic) direct sum of $\left\{W_{\pi}: \pi \in \Pi\right\}$ is a norm-dense, unital *-subalgebra (denoted by $\mathcal{C}_{0}$ from now on) of $\mathcal{C}$ on which $\alpha$ restricts to an algebraic co-action of $\mathcal{Q}_{0}$. We shall call $\mathcal{C}_{0}$ the Peter-Weyl subalgebra for the action $\alpha$.

An action $\alpha$ on $\mathcal{C}$ is called faithful if the $*$-subalgebra generated by $\{(\omega \otimes \mathrm{id})(\alpha(b))\}$, where $b \in \mathcal{C}$ and $\omega$ varying over the set of bounded linear functionals on $\mathcal{C}$, is dense in $\mathcal{Q}$. This is equivalent to the norm-density of the algebra generated by matrix coefficients of the irreducible representations $\pi$ for which $W_{\pi}$ is nonzero.

Given an action $\alpha$, we define $\alpha_{r}=\left(\mathrm{id} \otimes \pi_{r}\right) \circ \alpha$ and call it the reduced action. If the Haar state is faithful on $\mathcal{Q}$, we have $\alpha=\alpha_{r}$. Moreover, it is clear that the injectivity of $\alpha_{r}$ implies that of $\alpha$.

REMARK 2.3. A word of caution: our definition of a reduced action differs from that of many other authors, for example, [19, [15] and [4]. In fact, the term 'reduced' in our sense refers to the action by the reduced version of the quantum group, whereas the articles cited above considered a reduced version of the algebra, i.e. a suitable quotient of the algebra, on which the CQG acts.

Definition 2.4. We call an action $\alpha$ of a CQG $\mathcal{Q}$ on a unital $C^{*}$-algebra $\mathcal{C}$ to be implemented by a unitary representation $U$ of $\mathcal{Q}$ in $\mathcal{H}$, say, if there is a faithful representation $\pi: \mathcal{C} \rightarrow \mathcal{B}(\mathcal{H})$ such that $U(\pi(x) \otimes 1) U^{*}=(\pi \otimes$ id $)(\alpha(x))$ for all $x \in \mathcal{C}$.

It is clear that if an action is implemented by a unitary representation then it is one-to-one. In fact, as $U_{r}:=\left(\mathrm{id} \otimes \pi_{r}\right)(U)$ gives a unitary representation of $\mathcal{Q}_{r}$ in $\mathcal{H}$ and the 'reduced action' $\alpha_{r}:=\left(\mathrm{id} \otimes \pi_{r}\right) \circ \alpha$ of $\mathcal{Q}_{r}$ is also implemented by the unitary 
representation $U_{r}$, it follows that even $\alpha_{r}$ is one-to-one. We see below that the injectivity of $\alpha_{r}$ actually equivalent to implementability by a unitary representation.

LEMMA 2.5. Given an action $\alpha$ of $\mathcal{Q}$ on a unital separable $C^{*}$-algebra $\mathcal{C}$, the following are equivalent:

(a) There is a faithful positive functional $\phi$ on $\mathcal{C}$ which is invariant with respect to $\alpha$, i.e. $(\phi \otimes \mathrm{id})(\alpha(x))=\phi(x) 1_{\mathcal{Q}}$ for all $x \in \mathcal{C}$.

(b) The action is implemented by some unitary representation.

(c) The reduced action $\alpha_{r}$ of $\mathcal{Q}_{r}$ is injective.

Proof. If (a) holds, we consider $\mathcal{H}$ to be the GNS space of the faithful positive functional $\phi$. The GNS representation $\pi$ is faithful, and the linear map $V$ defined by $V(x):=\alpha(x)$ from $\mathcal{C} \subset \mathcal{H}=L^{2}(\mathcal{C}, \phi)$ to $\mathcal{H} \otimes \mathcal{Q}$ is an isometry by the invariance of $\phi$. Thus $V$ extends to $\mathcal{H}$ and it is easy to check that it induces a unitary representation $U$, given by $U(\xi \otimes q)=V(\xi) q$, which implements $\alpha$.

We have already argued $(\mathrm{b}) \Rightarrow(\mathrm{c})$, and finally, if (c) holds, we choose any faithful state $\tau$ (say) on the separable $C^{*}$-algebra $\mathcal{C}$ and take $\phi(x)=(\tau \otimes h)\left(\alpha_{r}(x)\right)$, which is faithful as $h$ is faithful on $\mathcal{Q}_{r}$ and $\alpha_{r}$ is injective. It can easily be verified that $\phi$ is $\alpha$-invariant on the dense subalgebra $\mathcal{C}_{0}$ mentioned before, and hence on the whole of $\mathcal{C}$.

We shall also need the following facts about actions on commutative $C^{*}$-algebras.

Proposition 2.6. If a $C Q G \mathcal{Q}$ acts faithfully on $C(X)$, where $X$ is a compact metrizable space, then $\mathcal{Q}$ is separable and it is also of Kac type, i.e. $\kappa^{2}=\mathrm{id}$ and the Haar state is tracial. In this case, $\kappa$ is norm-bounded on $\mathcal{Q}_{r}$.

Proof. Note that $X$ is second countable and hence $C(X)$ is separable. Choose a countable dense set of points $\left\{x_{i}: i=1,2, \ldots\right\}$ and a countable norm-dense subset $\left\{f_{n}: n=\right.$ $1,2, \ldots\}$ of $C(X)$. It follows from faithfulness of the action $\alpha$ (say) that $\mathcal{Q}$ is generated as a $C^{*}$-algebra by the countable set $\left\{\alpha\left(f_{n}\right)\left(x_{i}\right): i, n=1,2, \ldots\right\}$, hence it is separable.

For the proof of the Kac conditions, we refer to [11] as well as [7]. It is easy to see that condition (1) of Theorem 3.17 of [11] (Theorem 3.23 in the arXiv version) is trivially satisfied for commutative $C^{*}$-algebras. The norm-boundedness of $\kappa$ can easily be argued using the results of [23]. In fact, this has been done in [7] (see Proposition 2.1 and Lemma 2.2 of that paper).

3. Smooth actions are injective. Let $M$ be a compact smooth manifold. Let us recall the definition of smooth CQG action on it from [10].

Definition 3.1. We say that an action $\alpha$ of a CQG $\mathcal{Q}$ on $C(M)$ is smooth if $\alpha$ maps $C^{\infty}(M)$ into $C^{\infty}(M, \mathcal{Q})$ and $\operatorname{Sp} \alpha\left(C^{\infty}(M)\right)(1 \otimes \mathcal{Q})$ is dense in $C^{\infty}(M, \mathcal{Q})$ in the Fréchet topology. In this case, we will also say that $\alpha$ is a smooth action of $\mathcal{Q}$ on $M$.

THEOREM 3.2. If $\mathcal{Q}$ has a faithful smooth action $\alpha$ on $C^{\infty}(M)$, where $M$ is a compact smooth manifold, then for every fixed $x \in M$ there is a well-defined, *-homomorphic map $\epsilon_{x}$ from the unital $*$-subalgebra $\mathcal{Q}_{x}^{\infty}:=\left\{\alpha_{r}(f)(x): f \in C^{\infty}(M)\right\}$ of $\mathcal{Q}_{r}$ to $\mathbb{C}$ satisfying $\epsilon_{x}\left(\alpha_{r}(f)(x)\right)=f(x)$ for all $f \in C^{\infty}(M)$. 
Proof. Adapting the arguments of [17] and [1, we can get a Fréchet dense subalgebra $\mathcal{C}_{0}$ of $C^{\infty}(M)$ on which $\alpha$ restricts to an algebraic co-action of $\mathcal{Q}_{0}$. For example, $\mathcal{C}_{0}$ may be chosen as the analogue of the Peter-Weyl subalgebra in the sense of [1]. Replacing $\mathcal{Q}$ by $\mathcal{Q}_{r}$ we can assume without loss of generality that the Haar state of $\mathcal{Q}$ is faithful and $\alpha=\alpha_{r}$. In this case $\mathcal{Q}$ will has a bounded antipode $\kappa$ (by Proposition 2.6. Let $\alpha_{x}: C^{\infty}(M) \rightarrow \mathcal{Q}_{x}^{\infty}$ be the map defined by $\alpha_{x}(f):=\alpha(f)(x)$. It is clearly continuous with respect to the Fréchet topology of $C^{\infty}(M)$ and hence the kernel $\mathcal{I}_{x}$ (say) is a closed ideal, so that the quotient, which is isomorphic to $\mathcal{Q}_{x}^{\infty}$, is a nuclear space. Let us consider $\mathcal{Q}_{x}^{\infty}$ with this topology and then by nuclearity, the projective and injective tensor products of $\mathcal{Q}_{x}^{\infty}$ with $\mathcal{Q}$ (viewed as a Banach space, which is separable by Proposition 2.6 coincide. The multiplication map $m: \mathcal{Q}_{x}^{\infty} \otimes_{\text {alg }} \mathcal{Q} \rightarrow \mathcal{Q}$ extends to a continuous map (to be denoted by $m$ again) on $\mathcal{Q}_{x}^{\infty} \hat{\otimes} \mathcal{Q}$. It follows from the relation (id $\left.\otimes \Delta\right) \circ \alpha=(\alpha \otimes$ id) $\circ \alpha$ that $\Delta\left(\alpha_{x}(f)\right)=\left(\alpha_{x} \otimes \mathrm{id}\right)(\alpha(f))$, i.e. $\Delta$ maps $\mathcal{Q}_{x}^{\infty}$ to $\left(\alpha_{x} \otimes \operatorname{id}_{\mathcal{Q}}\right)\left(C^{\infty}(M) \hat{\otimes} \mathcal{Q}\right) \subseteq \mathcal{Q}_{x}^{\infty} \hat{\otimes} \mathcal{Q}$. Thus, the composite map $\beta:=m \circ($ id $\otimes \kappa) \circ \Delta: \mathcal{Q}_{x}^{\infty} \rightarrow \mathcal{Q}$ is continuous. Clearly, this map coincides with $\epsilon(\cdot) 1_{\mathcal{Q}}$ on the Fréchet-dense subalgebra $\mathcal{Q}_{x}^{0}$ (say) of $\mathcal{Q}_{x}^{\infty}$ spanned by elements of the form $\left\{\alpha(f)(x): f \in \mathcal{C}_{0}\right\}$. By the continuity of $\beta$, it follows that the range of $\beta$ is $\mathbb{C} 1_{\mathcal{Q}}$. Moreover, as $\beta(\alpha(f)(x))=f(x) 1_{\mathcal{Q}}$ for all $f \in \mathcal{C}_{0}$, and $\beta$ and $\alpha$ are Fréchet-continuous, we conclude that $\beta(\alpha(f))=f(x) 1_{\mathcal{Q}}$ for all $f \in C^{\infty}(M)$. Hence we can define $\epsilon_{x}$ by setting $\epsilon_{x}(\cdot) 1_{\mathcal{Q}}=\beta(\cdot)$. Clearly, $\epsilon_{x}(f)=f(x)$ for all $f \in C^{\infty}(M)$. This completes the proof of the theorem.

COROLlary 3.3. For any smooth action $\alpha$ on $C^{\infty}(M)$, the reduced action $\alpha_{r}$ is injective on $C(M)$.

Proof. Consider the $C^{*}$ algebra $\left(\mathcal{Q}_{1}\right.$, say) generated by the matrix coefficients $t_{i j}^{\pi}$ 's of the irreducible representations $\pi$ of $\mathcal{Q}$ for which the spectral subspace $W_{\pi}$ for $\alpha$ corresponding to $\pi$ is nonzero. It is easy to see that the restriction of the co-product of $\mathcal{Q}$ to $\mathcal{Q}_{1}$ leaves it invariant and makes it a CQG which acts faithfully on $C(M)$. Thus, replacing $\mathcal{Q}$ by $\mathcal{Q}_{1}$, we may assume that $\alpha$ is faithful. If $\alpha_{r}(f)=0$ for $f \in C^{\infty}(M)$ then for each $x$, applying $\epsilon_{x}$ obtained by Theorem 3.2 , we conclude $f(x)=0$ for all $x$, hence $f=0$. This proves the injectivity of the restriction of $\alpha_{r}$ to $C^{\infty}(M)$. Now, we have to prove its injectivity on the whole of $C(M)$. To this end, consider any positive Borel measure $\mu$ of full support on $M$, with $\phi_{\mu}$ being the positive functional obtained by integration with respect to $\mu$. Let $\psi:=\left(\phi_{\mu} \otimes h\right) \circ \alpha_{r}$ be the positive functional which is clearly $\alpha_{r}$-invariant and faithful on $C^{\infty}(M)$, i.e. $\psi(f)=0, f \in C^{\infty}(M)$ and $f$ nonnegative implies $f=0$. But by Riesz Representation Theorem there is a positive Borel measure $\nu$ such that $\psi(f)=\int_{M} f d \nu$. We claim that $\nu$ has full support, hence $\psi$ is faithful also on $C(M)$. This will complete the proof of the corollary by Lemma 2.5. Indeed, for any nonempty open subset $U$ of $M$ there is a nonzero positive $f \in C^{\infty}(M)$, such that $0 \leq f \leq 1$, and the support of $f$ is contained in $U$. By faithfulness of $\psi$ on $C^{\infty}(M)$ we get $0<\psi(f)=\int_{U} f d \nu \leq \nu(U)$.

As the injectivity of $\alpha_{r}$ clearly implies that of $\alpha$, we also get the following:

COROLlary 3.4. Any faithful smooth action of a CQG on a compact smooth manifold is injective. 
REMARK 3.5. In a recent work [8], Goswami has proved that any CQG which admits a faithful smooth action on a compact connected smooth manifold must be isomorphic to $C(G)$ for some group $G$ and the CQG action becomes a $G$-action. Hence any such action is injective. However, the injectivity result of the present note was used to prove the main result of [8], so we cannot use [8] to prove Corollary 3.3. Moreover, the results of this paper are applicable to a possibly disconnected manifold.

4. Smooth action on Rieffel deformation. Let us now consider CQG actions on noncommutative $C^{*}$-algebras. Rieffel deformation (see [18]) is a well-known and very useful procedure to obtain interesting noncommutative $C^{*}$-algebras from the commutative ones. In particular, given a smooth compact manifold $M$ equipped with the action of a compact abelian group $T \cong \mathbb{T}^{n}$, one can construct a family of (typically noncommutative) $C^{*}$-algebras $C(M)_{\theta}$ indexed by $n \times n$ skew symmetric matrices $\theta$. There is a similar procedure (see [21, [14] and the references therein) for deforming a CQG with some toral quantum subgroup of rank $n$ inducing an action of a torus of rank $2 n$ combining left and right actions by the elements of the $n$-toral subgroup. In this case, one gets a CQG by retaining the same co-algebra structure as the original one but changing the algebra structure. This will be called the Rieffel-Wang-Kasprzak (RWK) deformation of $G$.

Let $\mathcal{A}_{\theta}$ be the noncommutative $n$-torus, which is the universal $C^{*}$-algebra generated by unitaries $U_{1}, \ldots, U_{n}$ satisfying the commutation relations $U_{j} U_{k}=\exp \left(2 \pi i \theta_{j k}\right) U_{k} U_{j}$, where $\theta=\left(\left(\theta_{j k}\right)\right)$. Given a unital $C^{*}$-algebra $\mathcal{C}$ with a $\mathbb{T}^{n}$-action $\beta_{z}$ (say), the deformed $C^{*}$-algebra $\mathcal{C}_{\theta}$ can be described in two alternative ways: either in the original picture of Rieffel where one defines a new, twisted multiplication on the spectral algebra for the toral action and then considers appropriate $C^{*}$-completion, or as in [3], identifying $\mathcal{C}_{\theta}$ with the fixed point subalgebra $\left(\mathcal{C} \otimes \mathcal{A}_{\theta}\right)^{\beta \otimes v^{-1}}$ where $v_{z}$ denotes the canonical toral action on $\mathcal{A}_{\theta}$ satisfying $v_{z}\left(U_{i}\right)=z_{i} U_{i}$ for all $i$. We also have a 'dual' $T$-action on $\mathcal{C}_{\theta}$ which is the restriction of $(\mathrm{id} \otimes v)$ on $\mathcal{C} \otimes \mathcal{A}_{\theta}$.

Given a CQG $\mathcal{Q}$ and a quantum subgroup of $\mathcal{Q}$ isomorphic with $T=\mathbb{T}^{n}$, with the corresponding surjective CQG morphism $\pi: \mathcal{Q} \rightarrow C(T)$, we can define left and right $\mathbb{T}^{n}$-actions $\chi_{z}^{l}, \chi_{z}^{r}$ (say) respectively, by setting $\chi_{z}^{l}=\left(\operatorname{id} \otimes\left(\operatorname{ev}_{z} \circ \pi\right)\right) \circ \Delta$ and $\chi_{z}^{r}=\left(\left(\mathrm{ev}_{z} \circ \pi\right) \otimes \mathrm{id}\right) \circ \Delta$. Using this, we have a $\mathbb{T}^{2 n}$-action $\chi_{z, w}=\chi_{z}^{l} \chi_{w}^{r}$ on $\mathcal{Q}$ and the corresponding deformed CQG is the $C^{*}$-algebra $\mathcal{Q}_{\theta \oplus(-\theta)}$.

We have the following from Theorem 3.11 of [2].

LEmma 4.1. Let $\mathcal{C}$ be a unital $C^{*}$-algebra equipped with a $\mathbb{T}^{n}$-action given by $*$-automorphism $\beta_{z}, \mathcal{Q}$ be a reduced $C Q G$ with an action $\alpha$ of $\mathcal{Q}$ on $\mathcal{C}$ and a quantum subgroup of $\mathcal{Q}$ isomorphic with $\mathbb{T}^{n}$ as above (with the corresponding morphism $\pi$ ) satisfying $\beta_{z}:=$ $\left(\mathrm{id} \otimes\left(\mathrm{ev}_{z} \circ \pi\right)\right) \circ \alpha$. Then we have an action $\alpha_{\theta}$ of $\mathcal{Q}_{\tilde{\theta}}(\tilde{\theta}=\theta \oplus(-\theta))$ on $\mathcal{C}_{\theta}$. Here the deformation of $\mathcal{C}$ is taken with respect to the action $\beta$.

Consider now $\mathcal{C}=C(M)$, where $M$ is a compact smooth manifold equipped with a $\mathbb{T}^{n}$ action, which also induces a $\mathbb{T}^{n}$ action $(\operatorname{say} \beta)$ on $C(M)$. Let $\gamma=\beta \otimes v^{-1}$ as before and let us call the subalgebra $C^{\infty}(M)_{\theta} \equiv C^{\infty}\left(M, \mathcal{A}_{\theta}\right)^{\gamma} \subset C\left(M, \mathcal{A}_{\theta}\right)^{\gamma} \equiv\left(C(M) \otimes \mathcal{A}_{\theta}\right)^{\gamma}=C(M)_{\theta}$ the 'smooth subalgebra' and call an action $\alpha$ on $C(M)_{\theta}$ by a CQG $\mathcal{Q}$ to be smooth if it maps the above smooth subalgebra into $C^{\infty}\left(M, \mathcal{A}_{\theta} \otimes \mathcal{Q}\right)^{(\gamma \otimes \text { id })}$ and the linear span of 
$\left\{\alpha(F)(1 \otimes q): F \in C^{\infty}\left(M, \mathcal{A}_{\theta}\right)^{\gamma}, q \in \mathcal{Q}\right\}$ is dense in $C^{\infty}\left(M, \mathcal{A}_{\theta} \otimes \mathcal{Q}\right)^{(\gamma \otimes \mathrm{id})}$. Now, we can state and prove the following.

THEOREM 4.2. Let $M$ be as above and let $\alpha$ be a smooth action of a $C Q G$ on $C(M)_{\theta}$ in the above sense. Moreover, assume that there is a quantum subgroup of $\mathcal{Q}$ isomorphic with $T=\mathbb{T}^{n}$, given by a surjective $C Q G$ morphism $\pi: \mathcal{Q} \rightarrow C(T)$ such that $\left(\mathrm{id} \otimes\left(\mathrm{ev}_{z} \circ \pi\right)\right) \circ \alpha$ coincides with the canonical 'dual' $T$-action on $C(M)_{\theta}$. Then the action (in fact, the reduced one too) is injective.

Proof. We only very briefly sketch the proof. As before, assume without loss of generality that the CQG is reduced. It follows from the proof of Theorem 3.11 of 2 that the action $\alpha_{-\theta}$ of $\mathcal{Q}_{\tilde{\theta}}$ on $\left(C(M)_{\theta}\right)_{-\theta} \cong C(M)$ is smooth. We note that the word 'smooth' in the statement of Theorem 3.11 of [2] is used in a sense weaker than ours: it only means the invariance of the smooth algebra there. However, there is a canonical Fréchet-dense subalgebra (e.g. the analogue of the Peter-Weyl subalgebra) of $C^{\infty}(M)_{\theta}$ for the action $\alpha$, on which $\mathcal{Q}_{0}($ co)acts algebraically and which can be identified as a vector space with a Fréchet-dense subalgebra of $C^{\infty}(M)$ on which the deformed action (which is the same as $\alpha$ as a linear map on this space) $\alpha_{-\theta}$ is algebraic. From this, the Podles type density condition follows, i.e. $\alpha_{-\theta}$ is smooth in our sense. Hence it is injective by Corollary 3.3. Moreover, by that corollary and Theorem 2.5. we get a unitary representation of $\mathcal{Q}_{\tilde{\theta}}$ which implements $\alpha_{-\theta}$. But by the generalities of RWK (or, more general cocycle-twisted) deformation of CQG as in the Chapter 7 of [9], we conclude that $\alpha=\left(\alpha_{-\theta}\right)_{\theta}$ is unitarily implemented too, where the corresponding Hilbert space and unitary essentially remain the same. In particular, $\alpha$ is injective.

REMARK 4.3. In [14], it is shown that the Rieffel deformations of classical compact groups are co-amenable, hence actions by such deformed compact quantum groups are always injective. In combination with [8], this will prove Theorem 4.2 in the special case when $M$ is assumed to be connected. However, Theorem 4.2 remains valid even when $M$ is disconnected.

Acknowledgments. The first author would like to recall his fond memories of interaction with Prof. Paul Baum on several occasions: ICTP (Trieste), TIFR (India) and also in the conference in the Fields Institute in 2016. Although there was no discussion on the particular topic of this note, the first author is grateful to him for encouragement and suggestions regarding Baum-Connes' conjecture for quantum groups and other problems related to quantum symmetry on operator algebras. The authors wish him a long, healthy and creative life.

\section{References}

[1] P. F. Baum, K. De Commer, P. M. Hajac, Free actions of compact quantum groups on unital $C^{*}$-algebras, Doc. Math. 22 (2017), 825-849.

[2] J. Bhowmick, D. Goswami, Quantum isometry groups: examples and computations, Comm. Math. Phys. 285 (2009), 421-444. 
[3] A. Connes, M. Dubois-Violette, Noncommutative finite-dimensional manifolds. I. Spherical manifolds and related examples, Comm. Math. Phys. 230 (2002), 539-579.

[4] K. De Commer, Actions of compact quantum groups, in: Topological Quantum Groups, Banach Center Publ. 111, Polish Acad. of Sci. Inst. Math., Warsaw, 2017, 33-100.

[5] V. G. Drinfeld, Quantum groups, in: Proceedings of the International Congress of Mathematicians (Berkeley, 1986), Amer. Math. Soc., Providence, RI, 1987, 798-820.

[6] V. G. Drinfeld, Quasi-Hopf algebras, Algebra i Analiz 1 (1989), no. 6, 114-148; English transl.: Leningrad Math. J. 1 (1990), 1419-1457.

[7] D. Goswami, Quantum symmetries and quantum isometries of compact metric spaces, arXiv: 0811.0095

[8] D. Goswami, Non-existence of genuine (compact) quantum symmetries of compact, connected smooth manifolds, Adv. Math. 369 (2020), 107181.

[9] D. Goswami, J. Bhowmick, Quantum Isometry Groups, Infosys Sci. Found. Ser., Springer, New Delhi, 2016.

[10] D. Goswami, S. Joardar, Non-existence of faithful isometric action of compact quantum groups on compact, connected Riemannian manifolds, Geom. Funct. Anal. 28 (2018), 146178.

[11] H. Huang, Invariant subsets under compact quantum group actions, J. Noncommut. Geom. 10 (2016), 447-469, arXiv version 1210.5782 .

[12] M. Jimbo, A q-difference analogue of $U(\mathfrak{g})$ and the Yang-Baxter equation, Lett. Math. Phys. 10 (1985), 63-69.

[13] M. Jimbo, Quantum R-matrix for the generalized Toda system, Comm. Math. Phys. 102 (1986), 537-547.

[14] P. Kasprzak, The Heisenberg-Lorentz quantum group, J. Noncommut. Geom. 4 (2010), $577-611$.

[15] H. Li, Compact quantum metric spaces and ergodic actions of compact quantum groups, J. Funct. Anal. 256 (2009), 3368-3408.

[16] A. Maes, A. Van Daele, Notes on compact quantum groups, Nieuw Arch. Wisk. (4) 16 (1998), 73-112.

[17] P. Podleś, Symmetries of quantum spaces, subgroups and quotient spaces of $S U(2)$ and SO(3) groups, Comm. Math. Phys. 170 (1995), 1-20.

[18] M. A. Rieffel, Deformation Quantization for Actions of $R^{d}$, Mem. Amer. Math. Soc. 106 (1993), no. 506.

[19] P. Sołtan, On actions of compact quantum groups, Illinois J. Math. 55 (2011), 953-962.

[20] F. Treves, Topological Vector Spaces, Distributions and Kernels, Academic Press, New York, 1967.

[21] S. Wang, Deformation of compact quantum groups via Rieffel's quantization, Comm. Math. Phys. 178 (1996), 747-764.

[22] S. L. Woronowicz, Compact matrix pseudogroups, Comm. Math. Phys. 111 (1987), 613-665.

[23] S. L. Woronowicz, Compact quantum groups, in: Symétries quantiques (Les Houches, 1995), North-Holland, Amsterdam, 1998, 845-884. 
